\title{
Obtención de una placa fotosensible como material de registro holográfico
}

\author{
C. GARCÍA ${ }^{1}$, I. PASCUAL ${ }^{1}$ Y A. FIMIA ${ }^{2}$ \\ Departamento Interuniversitario de Óptica, Universidad de Alicante, Apdo. 99, 03080 Alicante. \\ Dpto. de Ciencias Experimentales y Tecnología. División de Óptica. Universidad Miguel Hernández. Campus de Elche. Avda. del Ferrocarril, s/n, \\ 03202 Alicante.
}

En este artículo se presenta, en primer lugar, el procedimiento de obtención y depositado de películas fotosensibles secas utilizadas como material de registro holográfico, comparando el espesor y uniformidad de las placas obtenidas con dos métodos de depositado distinto, con un depositador manual y con un depositador automático.

En segundo lugar se analiza la influencia del espesor en el comportamiento del material como material de registro holográfico.

Palabras claves: fotopolímeros, hologramas, materiales de registro holográfico

Obtention of photosensitive films as holographic recording materials

In this article was showed the procedure of obtaining and depositing of photosensitive dry films uses as holographic recording materials. We compare the thickness and the uniformity of plates obtained with two different depositing method, with a manual depositor and an automatic depositor.

The influence of the thickness on the behaviour of the material as holographic recording material has been studied.

Keywords: Photopolymers, holograms, holographic recording materials

\section{INTRODUCCIÓN}

En los últimos años se han desarrollado diferentes sistemas fotopolimerizables como material de registro holográfico ${ }^{1}$. Estos sistemas poseen características tales como buena sensibilidad espectral y energética, alta resolución, alto rendimiento en difracción, buena relación señal-ruido, estabilidad temporal y procesado en tiempo real. Todas estas características los hacen útiles en aplicaciones tales como: almacenamiento óptico holográfico, fabricación de elementos ópticos holográficos, interferometría holográfica, etc.

En este trabajo se presenta el procedimiento de obtención y depositado de películas fotosensibles secas utilizadas como material de registro holográfico y se estudia, al mismo tiempo, la influencia del método de depositado en la uniformidad de la placa obtenida.

Por último se analiza la influencia del espesor en el comportamiento del material como material de registro holográfico.

El material utilizado es un fotopolímero basado en acrilamidas $^{2,3,4}$, está formado por acrilamida como monómero, trietanolamina como generador de radicales y eosina amarillenta como fotoiniciador todo ello en una matriz de polivinilalcohol.

\section{PROCEDIMIENTO EXPERIMENTAL}

Como se ha señalado anteriormente, se han empleado dos métodos de depositado, uno manual y otro automático. En cada método se trabaja con dos espesores iniciales, $500 \mu \mathrm{m}$ y $1000 \mu \mathrm{m}$. En la tabla I se muestra la composición de la disolución fotosensible utilizada, excepto la concentración de polivi-
TABLA I: COMPOSICIÓN DE LA DISOLUCIÓN FOTOPOLIMERIZABLE

\begin{tabular}{|l|l|}
\hline Acrilamida (AA) & $0.518 \mathrm{M}$ \\
\hline Trietanolamina (TEA) & $0.199 \mathrm{M}$ \\
\hline Eosina amarillenta (EA) & $2.5 \mathrm{e}^{-4} \mathrm{M}$ \\
\hline
\end{tabular}

nilalcohol (PVA), que varía dependiendo del espesor inicial. Para un espesor inicial de $500 \mu \mathrm{m}$ se añade PVA al 10\% de concentración; sin embargo, cuando se trabaja con $1000 \mu \mathrm{m}$ de espesor es necesario aumentar la concentración de PVA, ya que el PVA influye en la consistencia de la placa y en el espesor final, por lo que después de realizar varias pruebas, variando la concentración de PVA, se obtuvo que la concentración óptima de PVA cuando se trabaja con $1000 \mu \mathrm{m}$ es del $15 \%$.

Para obtener la película seca, se deposita la disolución sobre un vidrio y se mantiene en la oscuridad en condiciones normales $\left(\mathrm{T} \approx 20^{\circ} \mathrm{C}, \mathrm{RH} \approx 50 \%\right.$ ). Uno de los problemas que se plantea es la obtención de placas con un espesor uniforme. En nuestro caso, las placas se utilizan para almacenar redes holográficas donde el espesor es un parámetro fundamental en el comportamiento del material. Según la teoría de Kogelnik $^{5}$, el espesor determina la evolución temporal del rendimiento en difracción. La variación en el espesor de este material fotopolimerizable, con la misma composición química, produce cambios en la forma de las curvas de rendimiento en difracción frente al tiempo y en la posición del máximo rendimiento en difracción. Por otra parte el espesor va a influir en la sensibilidad del material, aumentando a medida que éste aumenta. 


\section{RESULTADOS}

En este trabajo se comparan los resultados obtenidos al depositar placas de fotopolímero con un depositador manual y con un depositador automático. La cantidad de disolución que se deposita, así como el tamaño del vidrio utilizado depende del depositador que se utilice.

El depositador manual es un dispositivo "TLC Plate Coater", suministrado por Camag, consiste en una base y un soporte. El soporte se acopla sobre la base y se añade en él la disolución. El vidrio, de $20 \times 40 \mathrm{~cm}^{2}$, donde se deposita la disolución, se hace deslizar, empujado manualmente por otro cristal, entre esta base y el soporte, quedando impregnado de la disolución con un determinado grosor, el cual viene ajustado por el propio soporte. Este depositador permite trabajar con espesores iniciales de 100, 200, $300,500,1000 \mu \mathrm{m}$. En las condiciones de humedad y temperatura en las que se trabaja, una vez seca la placa su espesor inicial disminuye en un $80 \%$ aproximadamente. Uno de los problemas que plantea este método de depositado en cuanto a la uniformidad de las placas obtenidas, es que no es posible controlar la velocidad de extensión, lo que hace que no se consiga repetibilidad entre unas placas y otras. Por otra parte, en una misma placa se observan gradientes de espesores entre unas zonas y otras. El espesor promedio obtenido para un espesor inicial de $500 \mu \mathrm{m}$ es de $90 \pm 20 \mu \mathrm{m}$ y para un espesor de $1000 \mu \mathrm{m}$ es de $116 \pm$ $2 \mu \mathrm{m}$ En las figuras 1 y 2 se muestra un mapa de espesores para una placa de $20 \times 40 \mathrm{~cm}^{2}$ obtenida mediante el depositador manual con un espesor inicial de $500 \mu \mathrm{m}$ y $1000 \mu \mathrm{m}$ respectivamente.

El depositador automático, suministrado por NEURTEK, está formado por una base metálica sobre la que se coloca el vidrio, que en este caso es de $23 \times 40 \mathrm{~cm}^{2}$, y un soporte donde se colocan unas varillas que determinan el espesor de la placa. Al igual que el depositador manual permite obtener placas con distintos espesores iniciales, $100 \mu \mathrm{m}$, $200 \mu \mathrm{m}, 500 \mu \mathrm{m}$ y $1000 \mu \mathrm{m}$, pero en este caso si es posible controlar la velocidad con la que se extiende la disolución sobre el vidrio, pudiéndose realizar el depositado a distintas velocidades enumeradas de 1 a 10. La disolución se deposita sobre la placa y se extiende automáticamente mediante la varilla con una velocidad constante. Inicialmente se realizó un estudio sobre la uniformidad de las capas depositadas variando los espesores y las velocidades, encontrando que la velocidad óptima de depositado depende del espesor inicial utilizado. Se ha trabajado con espesores de $500 \mu \mathrm{m}$ y $1000 \mu \mathrm{m}$, al igual que con el depositador manual. En la figura 3 se muestra el espesor medio de la placa frente a la velocidad de depositado para los dos espesores utilizados. Se ha trabajado con velocidades 2, 3, 4 y 5, ya que con velocidades mayores se perdía disolución que se derramaba por los bordes. Cuando el espesor inicial es de $500 \mu \mathrm{m}$ se obtienen unos espesores que varían entre $70 \mu \mathrm{m}$ y $80 \mu \mathrm{m}$. En este caso, la velocidad óptima de depositado es de 3 , ya que esta velocidad es la que nos da un menor error de dispersión (el espesor promedio de la placa para esta velocidad es de $77 \pm 4 \mu \mathrm{m}$ ).

Cuando el espesor inicial es de $1000 \mu \mathrm{m}$ se obtienen unos espesores que varía entre 110 y $140 \mu \mathrm{m}$. En este caso, la velocidad óptima es de 2, ya que es la que menor error de dispersión nos da. El espesor promedio de la placa para la velocidad de 2 es de $116 \pm 4 \mu \mathrm{m}$. En las figuras 4 y 5 se representa el mapa de espesores correspondiente a las placas con espesor

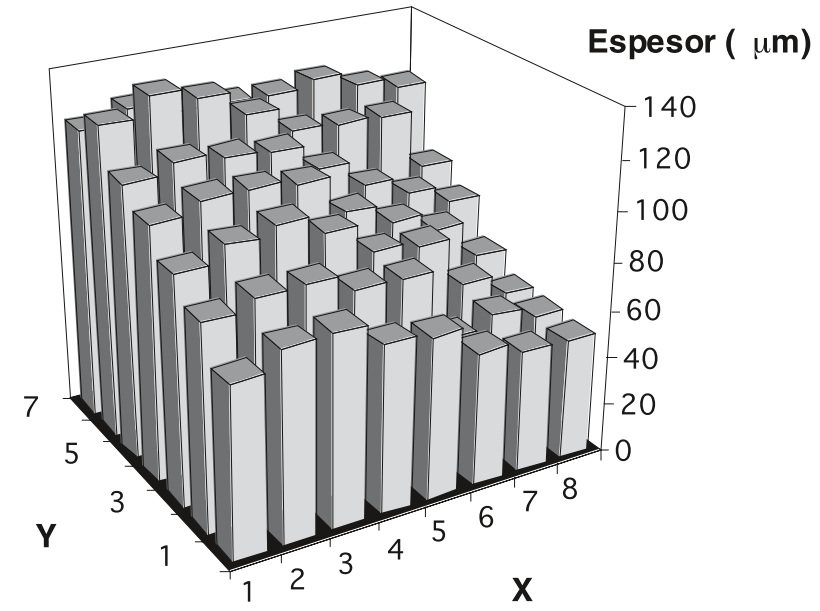

Figura 1. Mapa espesores de una placa con un espesor inicial de $500 \mu \mathrm{m}$ obtenida con el depositador manual.



Figura 2. Mapa espesores de una placa con un espesor inicial de $1000 \mu \mathrm{m}$ obtenida con el depositador manual.

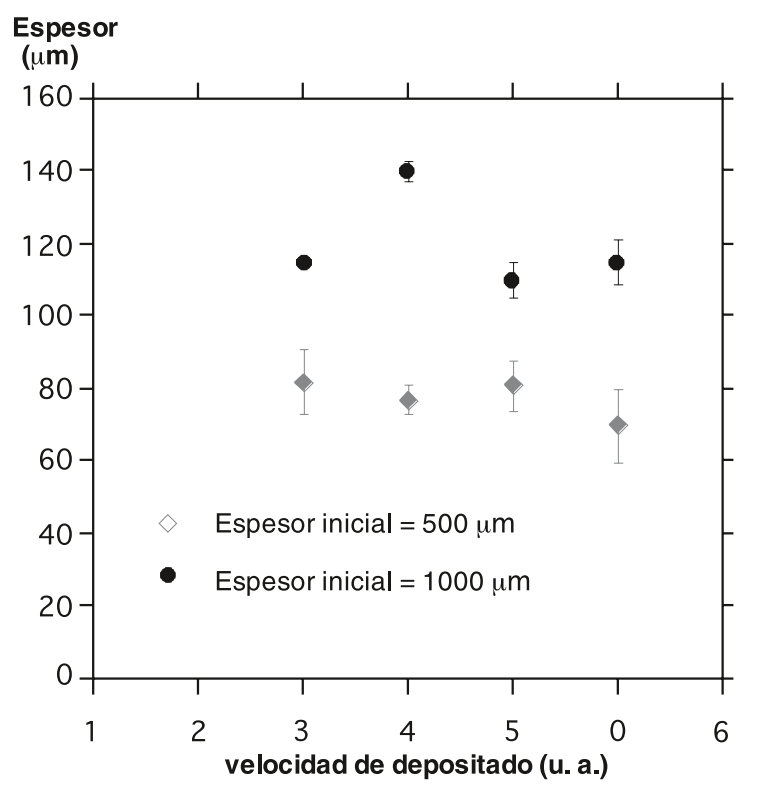

Figura 3. Velocidad de depositado frente al espesor. 
inicial de $500 \mu \mathrm{m}$ y $1000 \mu \mathrm{m}$, respectivamente, cada una de ellas obtenida con su velocidad óptima de depositado.

Si comparamos los resultados obtenidos con los dos depositadores (figuras 1, 2, 4 y 5) encontramos que la zona de placa uniforme para el depositador manual es menor, en ambos espesores y no siempre es la misma. La zona uniforme del depositador automático es mayor y corresponde siempre a la misma zona independientemente del espesor inicial. Con éste último podemos lograr una zona de espesor uniforme de $20 \times 25 \mathrm{~cm}^{2}$. Asimismo, existen mayores gradientes de espesor con el depositador manual que con el automático, aunque estas diferencias son mucho más significativas cuando se trabaja con un espesor inicial de $500 \mu \mathrm{m}$ que al trabajar con un espesor inicial de $1000 \mu \mathrm{m}$.

De todo esto se concluye que el depositador automático presenta ventajas frente al depositador manual en cuanto a uniformidad de la placa, el espesor es un parámetro fundamental a la hora de almacenar redes holográficas. Para almacenar las redes holográficas se utiliza el dispositivo experimental mostrado en la figura 6, utilizando un láser de Argon emitiendo a $514 \mathrm{~nm}$. El haz emergente se hace incidir sobre una lámina separadora, que nos permite obtener dos haces. Cada haz atraviesa un filtro y una lente, incidiendo sobre la placa como un haz plano. Ambos haces inciden en modo simétrico sobre el material de registro formando un ángulo de $16.8^{\circ}$ respecto a la normal, lo que nos permite almacenar redes holográficas con una frecuencia de 1125 líneas / $\mu \mathrm{m}$. Se trabaja con una relación de haces 1:1 y una intensidad incidente de 10 $\mathrm{mW} / \mathrm{cm}^{2}$. Para monitorizar en tiempo real las redes formadas se utiliza un láser de $\mathrm{He}-\mathrm{Ne}$, emitiendo a $633 \mathrm{~nm}$, que incide sobre la placa con el ángulo de Bragg $\left(20.9^{\circ}\right.$ respecto a la normal) La intensidad difractada y la intensidad transmitida son detectadas por dos detectores que están conectados a un PC, en el que se obtiene la evolución temporal de la intensidad difractada y la intensidad transmitida. Con estos datos puede obtenerse la evolución energética del rendimiento en difracción y en transmisión para las redes de difracción almacenadas.

Para evaluar la influencia del espesor en el comportamiento del material, se han realizado varias pruebas con distintos espesores. La figura 7a representa la evolución energética del rendimiento en difracción (RD), rendimiento en transmisión (RT) y suma de ambos (RD +RT) para un espesor de $55 \mu \mathrm{m}$. Las figuras $7 \mathrm{~b}$ y $7 \mathrm{c}$ representan las mismas curvas para unos espesores de $110 \mu \mathrm{m}$ y $210 \mu \mathrm{m}$ respectivamente. Como se ha señalado anteriormente, el espesor, de acuerdo con la teoría de Kogelnik, determina la evolución energética del rendimiento en difracción. Variaciones de espesor en este material con la misma composición química produce cambios en el comportamiento de este tipo de curvas Esto se observa al comparar las figuras 7a, 7b y 7c. Para el espesor de $55 \mu \mathrm{m}$, la curva de rendimiento en difracción alcanza su máximo valor y se mantiene constante. Al aumentar el espesor a $110 \mu \mathrm{m}$ se observa que la curva sube hasta el rendimiento en difracción máximo y decrece, en este caso la caída no es muy pronunciada. Si seguimos aumentando el espesor hasta $210 \mu \mathrm{m}$, se observa que al alcanzar el valor máximo de rendimiento en difracción la curva decae rápidamente.

De todo esto se deduce que cuando se trabaja con espesores bajos, del orden de $55 \mu \mathrm{m}$, el comportamiento del material de registro es muy bueno para su aplicación en la obtención de hologramas, pues una vez alcanzado el máximo rendimiento en difracción éste no decae, lo cual indica que hay poco ruido y por tanto pueden registrarse hologramas de gran calidad

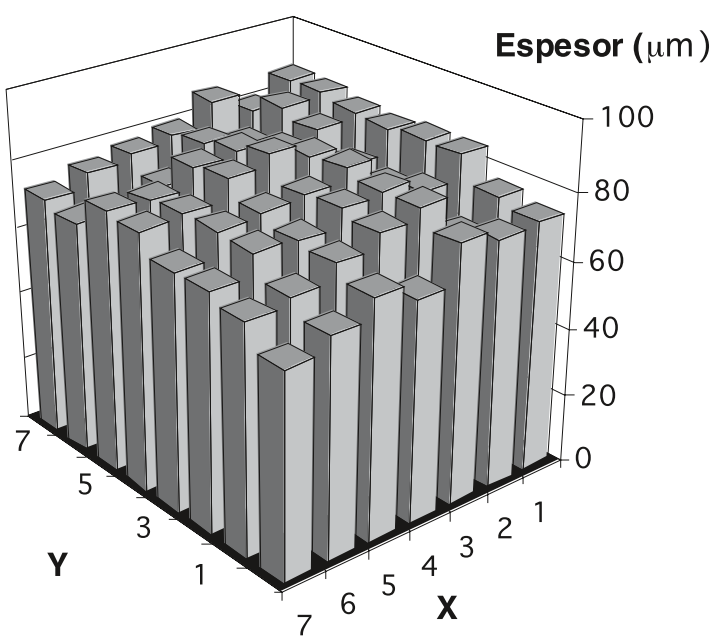

Figura 4. Mapa de espesores de una placa con un espesor inicial de $500 \mu \mathrm{m}$, obtenida con un depositador automático



Figura 5. Mapa de espesores de una placa con un espesor inicial de $1000 \mu \mathrm{m}$, obtenida con un depositador automático



Figura 6: Dispositivo experimental 
incluso con exposiciones elevadas. Si el espesor de las placas aumenta hasta un valor de $110 \mu \mathrm{m}$ las redes de difracción almacenadas una vez que han alcanzado el rendimiento en difracción máximo, comienzan a deteriorarse ya que éste decae aunque la caída es poco pronunciada y por lo tanto hay poco ruido almacenado. Es decir disponemos de una pequeña zona de exposición para la cuál el rendimiento del holograma y por tanto su calidad se mantiene constante.

Si el espesor de la capa de fotopolímero es del orden de las $210 \mu \mathrm{m}$, sólo se pueden almacenar redes holográficas para la exposición que nos permite alcanzar el máximo rendimiento en difracción, pues una vez alcanzado aparecen redes de ruido que hacen que se difunda mucha luz, por lo que tanto el rendimiento en difracción y el rendimiento en transmisión decrecen, así como la calidad del holograma.

El espesor también influye en la sensibilidad, se observa que al aumentar el espesor del material aumenta la sensibilidad, pasando de $300 \mathrm{~mJ} / \mathrm{cm}^{2}$ para un espesor de $55 \mu \mathrm{m}$ hasta $100 \mathrm{~mJ} / \mathrm{cm}^{2}$ para un espesor de $210 \mu \mathrm{m}$. En cuanto al rendimiento en difracción máximo alcanzado no hay grandes variaciones al aumentar el espesor, el rendimiento en difracción varía entre el $75 \%$ y el $90 \%$.

La principal diferencia en el comportamiento del material al aumentar el espesor es que aparecen muchas redes de ruido, por lo que el rendimiento al alcanzar su máximo valor decae rápidamente. Sin embargo si comparamos los puntos para los cuales se consigue máximo rendimiento en difracción vemos que el scattering no varía prácticamente con el espesor.

Esto se observa en la figura 8 donde se representa el scattering en el punto de rendimiento máximo y el scattering en el punto de rendimiento final frente al espesor. El espesor se ha variado desde $55 \mu \mathrm{m}$ hasta $210 \mu \mathrm{m}$. Se ha tomado como medida del scattering en un punto la diferencia entre la suma del haz transmitido y difractado en ese punto y el valor inicial.

$\mathrm{Al}$ aumentar el espesor el scattering final aumenta desde un $2 \%$ para un espesor de $55 \mu \mathrm{m}$ hasta el $80 \%$ para un espesor de $210 \mu \mathrm{m}$. Sin embargo el scattering en el punto de rendimiento máximo no supera el 10\% para ninguno de los espesores.

Esto plantea la utilización de este material en la fabricación de memorias holográficas. A. Pu and D. Psaltis ${ }^{6}$ han establecido una relación lineal entre la densidad de almacenamiento y el espesor del material, según la cual en un espesor de 200 $\mu \mathrm{m}$ se podrían almacenar $60 \mathrm{bits} / \mu \mathrm{m}^{2}$.

La zona óptima de trabajo para este espesor estaría por debajo de los $100 \mathrm{~mJ} / \mathrm{cm}^{2}$, más allá de esta exposición el rendimiento en difracción disminuye y aparecen muchas redes de ruido.

\section{CONCLUSIONES}

De todo esto se concluye que al comparar los dos sistemas de depositado, el depositador automático presenta ventajas frente al depositador manual en cuanto a uniformidad de la placa en espesor, lo que nos permite obtener una mayor repetibilidad en los resultados cuando almacenamos redes holográficas.

$\mathrm{Al}$ estudiar la influencia del espesor en el comportamiento del material obtenemos que variaciones de espesor en placas con la misma composición química producen cambios en el comportamiento de este tipo de curvas. Por otra parte a pesar de que al aumentar el espesor una vez alcanzado el rendimiento máximo la curva decrece se observa que en el punto de rendimiento máximo el scattering no varia, y el rendimiento en difracción se mantiene prácticamente constante.

Todas estas características permiten plantear la utilización

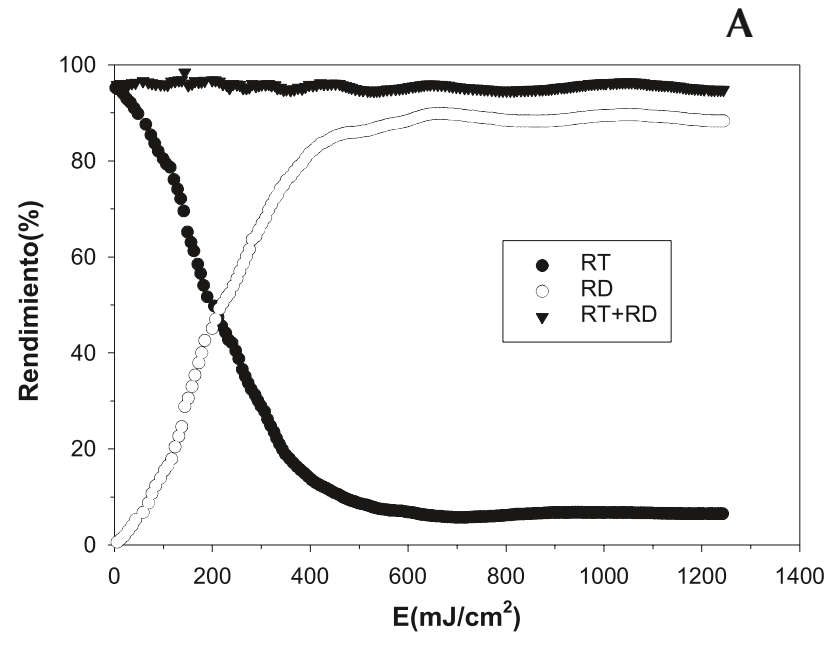

B

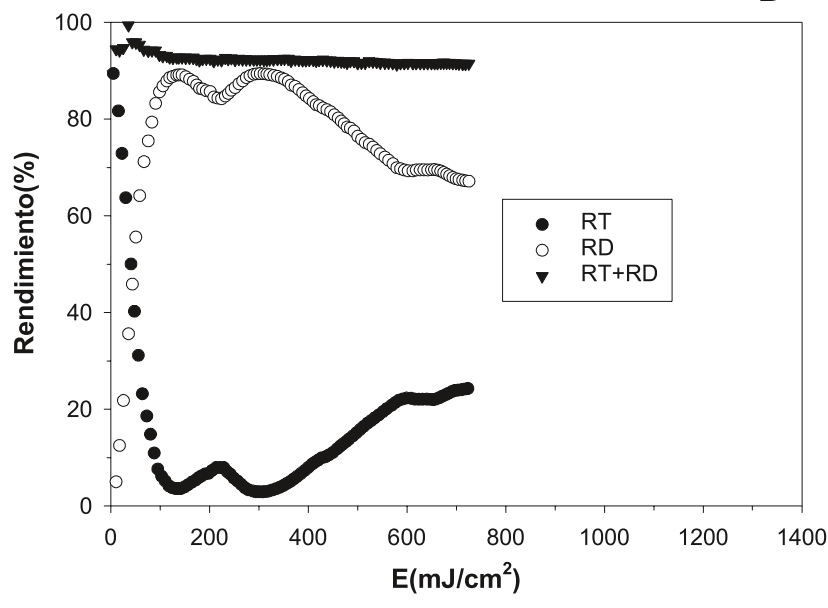

C

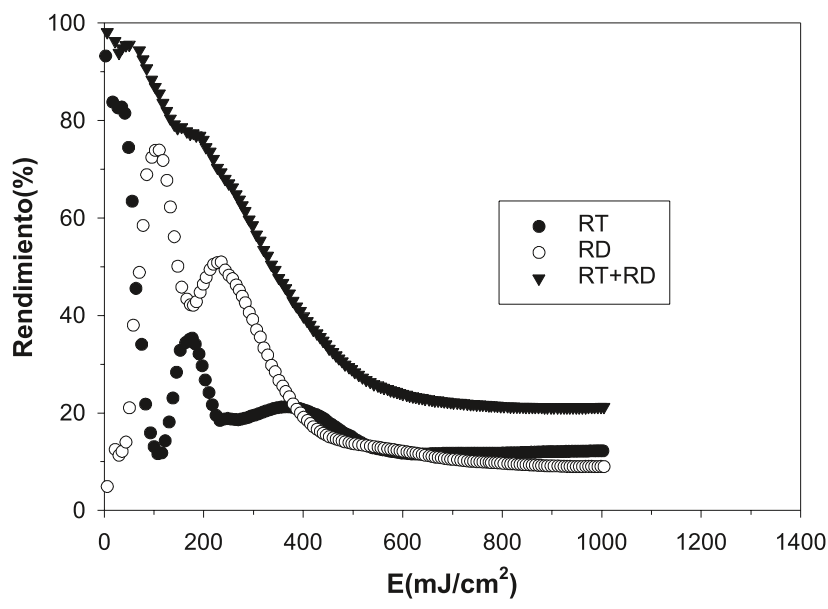

Figura 7: Rendimiento en difracción, rendimiento en transmitancia y suma de ambos para a )d =55 $\mu \mathrm{m}, \mathrm{b}) 110 \mu \mathrm{m}$ y c) $210 \mu \mathrm{m}$.

de este material en la fabricación de memorias holográficas. La zona óptima de comportamiento del material está por debajo de los $100 \mathrm{~mJ} / \mathrm{cm}^{2}$ para un espesor de $210 \mu \mathrm{m}$. En esta zona es posible almacenar hologramas con alto rendimiento en difracción y bajo scattering. 


\section{AGRADECIMIENTOS}

Este trabajo ha sido posible gracias a la financiación de la Comisión Interministerial de

Ciencia y Tecnología (C.I.C.Y.T.) del Ministerio de Educación y Ciencia, mediante el proyecto MAT 97-0705C02-02.

\section{BIBLIOGRAFÍA}

1. R. A. Lessard y G. Manivannan, "Holographic Recording Materials: An Overwiew", in Holographic Materials, SPIE 2405, 2-23 (1995).

2. C. García, I. Pascual and A. Fimia, "Contrast of diffuse object holograms in PVA-Acrylamide photopolymers: Real time measurements," in Holographic Materials V, SPIE 3638, 113-118 (1998).

3. S. Blaya, L. Carretero, R. Mallavia, A. Fimia, M. Ulibarrena y D. Levy, "Optimization of an Acrylamide-base dry film used for Holographic Recording," Appl. Opt. 37, pp. 7604-7610, (1998).

4. C. García, "Influencia de la intensidad en la calidad óptica de hologramas de objetos difusores almacenados en fotopolímeros,", Memoria de Investigación, Universidad de Valencia, (1998).

5. H. Kogelnik, "Coupled wave theory for thick hologram gratings", Bell. Sys. Tech. J. 48, 2909-2947 (1969).

6. Pu and D. Psaltis, "High-density recording in photopolymer-based holographic three-dimensional disks", Appl. Opt. 35, pp. 2389-2398, (1996).



Figura 8: Scattering en el punto de rendimiento máximo y scattering en el punto de rendimiento final frente al espesor. 